\title{
LOW LEVEL LASER THERAPY IN THE TREATMENT OF TEMPOROMANDIBULAR JOINT PAIN: A DOUBLE-BLIND STUDY
}

Hend Bahgat Mahmoud Thabet ${ }^{*}$, Hamida Refai* ${ }^{* *}$, Nermine Hassanin ${ }^{* * *}$ and Ahmed Abbas Zaki ${ }^{* * * *}$

\begin{abstract}
Aim of the Study: was to evaluate the effectiveness of low level laser therapy for the control of TMJ pain.

Materials and Methods: This study included 12 patients with clinical diagnosis of DDWR in a random and double blind research design. Patients, divided into study group and control (placebo) group submitted to treatment with LLL using Ga-As diode laser. Study group received 3 sessions per week during 4 consecutive weeks while placebo group received inactive laser using the same technique and schedule as study group.
\end{abstract}

Results: There were not statistically significant differences between both groups.

KEYWORDS: low intensity laser therapy, laser therapy, temporomandibular joint, temporomandibular disorder treatment.

\section{INTRODUCTION}

There is no standard therapeutic protocol for treatment of disc derangement with reduction (DDWR) of temporomandibular joint (TMJ). This could be attributed to lack of knowledge of their definite causes with subsequent evolution of many theories and treatment modalities leading to confusion.
There is a wide variety of surgical and nonsurgical treatment modalities for DDWR ${ }^{(1-3)}$. These modalities range from pharmacological to surgical and occasionally physical therapy is also used. The principal aim is to relieve pain and restore mandibular range of motion.

Being self-limiting, conservative treatments of DDWR has been advocated ${ }^{(4,5)}$. Low Level Laser

\footnotetext{
*B.D.S. 2002.Faculty of Oral and Dental Medicine, Cairo University. **Professor of Oral and Maxillofacial Surgery. Faculty of Oral and Dental Medicine, Cairo University. ***Associate Professor of Oral and Maxillofacial Surgery. Faculty of Oral and Dental Medicine, Cairo University. **** Associate Professor of Dental Laser Application. National Institute of Laser Enhanced Sciences(NILES), Cairo University.
} 
Therapy (LLLT) is one of the recent treatment modalities in the field of physiotherapy.

It is believed to have biostimulating, regenerative, analgesic and anti-inflammatory effects ${ }^{(6-7)}$. The clinical efficacy of LLLT has been studied with contradictory results ${ }^{(8-9)}$. Some studies found LLLT to be superior to placebo therapy, others found no significant differences between both ${ }^{(8,10)}$.

It has been reported that the use of inadequate power or energy density could cause undesirable effects ${ }^{(11)}$. To avoid heterogeneity in patient sample, this study was designed in double blind placebo controlled clinical trial to evaluate LLLT efficacy in management of TMJ pain in patients with DDWR.

\section{PATIENTS AND METHODS}

Twelve patients with DDWR participated in the study. 2 males and 10 females. Their ages ranged from 13-39 years. They were recruited from outpatient clinic, Oral Surgery Department, Faculty of Oral and Dental Medicine, Cairo University.

\section{Study design:}

Randomization and assignment of patients to one of 2 equal treatment groups were done by a person not involved in the study. The study has been approved by the Research Ethics Committee at the Faculty of Oral and Dental Medicine, Cairo University.

\section{Grouping of patients}

After written consent, the selected patients were randomly assigned to either the laser or placebo group.

\section{LASER GROUP}

Each joint received active application of LLL using Ga-As (Gallium-Arsenide) diode laser in 3 sessions per week during 4 consecutive weeks.

\section{PLACEBO GROUP}

The same technique and schedule as laser group but with inactive laser.

\section{Preoperative evaluation}

Included history, and clinical examination. Data for each patient were collected in his or her own questionnaire.

\section{TMJ EXAMINATION}

\section{A) TMJ FUNCTION}

- Maximal inter-incisal opening(MIO) measured in $\mathrm{mm}$.

- Presence of mandibular deviation during mouth opening.

- Maximum protrusive movement measured in $\mathrm{mm}$.

- Maximum lateral excrusion measured in $\mathrm{mm}$.

\section{B) TMJ PALPATION}

TMJ was examined by palpation via preauricular and meatal approaches to determine:

- Presence of TMJ tenderness or pain.

- Translatory movement of condyle during different mandibular movements.

- Joint sounds.

\section{Laser treatment}

Anatomic landmarks were located by asking the patient to open widely to allow drawing of the articular fossa and then close lightly on the posterior teeth to draw the condyle within the glenoid fossa.

QUANTA C Laser Unit, QUANTA SYSTEM spa, Italy, producing diode gallium arsenide ( $\mathrm{Ga} A s)$ laser was used in this study (Fig.1). Semi-conductor Ga As laser of wavelength 980 nanometers, output power of 0.2 Watt, total energy of 12 Joules and exposure time of 60 seconds. LLLT was achieved 
through light and direct contact of laser probe on the affected TMJ, anterior, posterior and lateral to the condyle. The treatment was performed 3 times per week for 4 consecutive weeks. For the placebo group, the device was adjusted for the same time and applied in the same manner but without power.

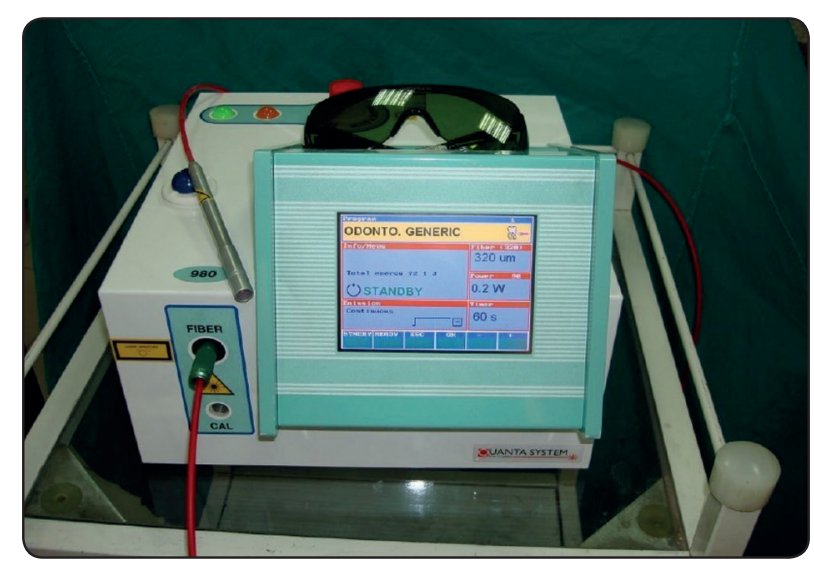

Fig. (1) QUANTA C Laser Unit

\section{Outcome measures}

The following outcome measures were clinically assessed and documented before and after $4^{\text {th }}, 8^{\text {th }}$, $12^{\text {th }}$ applications as well as two months after the last application.

Patients' subjective pain experience.

Maximum mouth opening (MMO).

The maximum lateral excursion.

\section{Statistical analysis:}

Microstat7 for windows statistical package (Microstat Co) was used for statistical analysis in this study. One-way ANOVA was used to evaluate effect of time in each group followed by calculating LSD (least significant difference) for paired comparisons of each interval. Independent student " $t$ " was used to compare both groups at each interval. Difference was considered statistically significant when $\mathrm{p}<0.05$.

\section{RESULTS}

\section{Subjective Findings}

\section{TMJ Pain at rest}

NRS was used to evaluate TMJ pain. There was statistically insignificant difference between both groups at all follow up intervals. In the laser group, the means of pain scores showed insignificant increase after the $4^{\text {th }}$ application followed by insignificant decrease until last interval. In the placebo group, the means of pain scores showed insignificant decrease during all intervals.

\section{TMJ pain during function}

There was statistically insignificant difference between both groups at all follow up intervals. In the laser group, the means of pain scores showed insignificant increase after the $4^{\text {th }}$ application followed by insignificant decrease until last interval. In the placebo group, the means of pain scores showed insignificant decrease after the $4^{\text {th }}$ and $8^{\text {th }}$ applications, while this decrease was statistically significant after the $12^{\text {th }}$ application.

\section{Objective Findings:}

\section{Pain on pressure (pp):}

There was statistically insignificant difference between both groups at all follow up intervals, except at preoperative and after the $4^{\text {th }}$ application laser group showed significant decrease in pain scores compared to placebo group. The means of pain scores in each group showed insignificant decrease after the $4^{\text {th }}$ and $8^{\text {th }}$ applications, while this decrease was statistically significant after the $12^{\text {th }}$ application and 2 months after last session.

\section{Maximum mouth opening (MMO):}

There was statistically insignificant difference between both groups at all follow up intervals except at preoperative interval whereas the mean of MMO in the laser group was statistically higher than 
that of the placebo group. In each group, there was insignificant increase in the means of MMO after the $4^{\text {th }}$ and $8^{\text {th }}$ applications followed by significant increase after the $12^{\text {th }}$ applications followed by insignificant decrease at the final interval.

\section{Lateral movement:}

There was statistically insignificant difference between both groups at all follow up intervals. In the laser group, the means of lateral movements showed insignificant increase after the $4^{\text {th }}$ and $8^{\text {th }}$ applications followed by significant increase after the $12^{\text {th }}$ application followed by insignificant decrease at the final interval. In the placebo group, the means of lateral movements showed insignificant increase after the 4th application followed by significant increase after the $8^{\text {th }}$ and $12^{\text {th }}$ applications followed by insignificant decrease at the final interval.

\section{Protrusive movement}

There was statistically insignificant difference between both groups at all follow up intervals. In the laser group, the means of protrusive movements showed insignificant increase during all follow up intervals. In the placebo group, the means of protrusive movements showed insignificant increase after $4^{\text {th }}$ and $8^{\text {th }}$ application followed by significant increase after the $12^{\text {th }}$ application followed by insignificant decrease at the final interval.

\section{DISCUSSION}

In the current study, laser and placebo groups showed significant decrease in pain scores during function and on pressure after the $12^{\text {th }}$ application. Also, by the end of study, pain on pressure scores was significantly decreased. Our findings are consistent with those of Maia et al ${ }^{(12)}$ who investigated the effect of LLLT on the pain levels in individuals with TMD in a systematic review of 14 studies and found that reduction in pain levels was reported in 13 studies.The analgesic effect of LLLT could be explained in part based on the findings of experimental and clinical studies. The improvements in TMJ pain during function, for placebo LLLT group were reported in other studies ${ }^{(8,10)}$. This finding emphasizes once again the power of the placebo effect that has been widely demonstrated in the treatment of TMD. Moreover, the self-limiting aspect of the TMD, with periods of remission of symptoms, may partly explain not only the response treatment of the placebo group, but also the pain reduction for the experimental group $^{(8)}$.

Improvement in the mandibular movements was observed in both groups throughout the study intervals. In the laser group, the improvement in MMO and lateral movements was significant after the $12^{\text {th }}$ application. While in the placebo group, the improvement in all the mandibular movements was significant after the $12^{\text {th }}$ application. The improvement in lateral and protrusive movements was also significant after the 8th application. The improvement in mandibular movements in the group treated with laser could not be attributed to its recognized analgesic effect as claimed by Núñez et al ${ }^{(13)}$. Since placebo group showed improvement in all the mandibular movements suggesting a role for the placebo effect in both groups. Contrary, Kulekcioglu et al (14) found that active and passive maximum mouth openings as well as lateral motion were significantly improved only in the active treatment group.

In the present study, comparison between the laser and placebo groups did not result in significant difference in pain scores on rest and function throughout the study intervals. Also, there were insignificant differences between the two groups in the improvement in all the mandibular movements. However, the laser group showed significant decrease in pain scores on pressure compared to placebo group preoperatively and after the 4th application. Interestingly, the evaluated parameters in both groups demonstrated high standard 
deviations, indicating significant variability in the data. Our findings are in general agreement with those of other investigators who found no significant differences between real and placebo groups. ${ }^{(8,10)}$. However, several studies ${ }^{(14,15)}$ found superiority of laser group over the placebo group.

There are several limitations of this study. First of all was the small sample size. Another limitation was variability as reflected by the preoperative significant differences between the two studied groups in pain on pressure and in MMO that may impact the results. Finally, the long term results of the therapy were not evaluated.

\section{CONCLUSION}

The Low level laser therapy seems to have no superiority over placebo laser application in reducing pain severity and in improving of range of mandibular motion.

\section{REFERENCES}

1. Carlsson GE. Long-term effect treatment of craniomandibular disorders. Cranio (1995)3:337- 342.

2. Carlsson GE. Epidemiology and treatment need for temporomandibular disorders. J Orofac Pain (1999) 13:295-306.

3. Chortis AG, Chorti AG, G Forrester and G Georgoudis. Therapeutic exercise in the management of ADD of the TMJ. Physical Therapy Reviews (2006) 11: 117-123.

4. Clark GT, Adachi NY and Dorman MR. Physical medicine procedures affect temporomandibular disorders: A review. J Am Dent Assoc (1990) 119: 121-151

5. Medlicott MS and Harris SR. A systematic review of the effectiveness of exercise, manual thrapy, electrotherapy, relaxation training, and biofeedback in the management of temporomandibular disorder. Phys Ther (2006) 86:955-973.
6. Walsh LJ. The current status of laser applications in dentistry. J Aust Dent (2003) 48:146-155.

7. Yu W, Naim JO and Lanzafame RJ. The effect of laser irradiation on the release of bFGF from 3T3 fibroblasts. Photochem Photobiol (1994) 59:167-170.

8. De Abreu Venancio, Camparis CM and Defa'tima Zanirato Lizarelli R. Low intensity laser therapy in the treatment of temporomandibular disorders: a double-blind study. J Oral Rehabil (2005) 32:800-807.

9. Bertolucci LE and Grey T. Clinical analysis of mid-laser versus placebo treatment of arthralgic TMJ degenerative joints. J Craniomand Pract (1995) 13:26-29.

10. Emshoff R, Bösch R, Pümpel E, Schöning H and Strobl H. Low-level laser therapy for treatment of temporomandibular joint pain: a double-blind and placebo-controlled trial. Oral Surg Oral Med Oral Pathol Oral Radiol Endod (2008) 105:452-456.

11. Van Breugel HHFI and Bar PR. Power density and exposure time of $\mathrm{HeNe}$ laser irradiation are more important than total energy dose in photobiomodulation of human fibroblast in vitro. Lasers in Medicine and Surgery (1992) 12: 528-537.

12. Maia MLM, Bonjardim LR, Quintans JSS, Ribeiro MAG, Maia LGM and Conti PCR. Effect of low-level laser therapy on pain levels in patients with temporomandibular disorders: a systematic review. J Appl Oral Sci (2012) 20(6):594-602.

13. Nunez SC, Garcez AS, Suzuki SS and Riberio MS. Management of Mouth Opening in Patients with Temporomandibular Disorders through Low-Level Laser Therapy and Transcutaneous Electrical Neural Stimulation. Photomed and Laser surg (2006) 24(1):45-49.

14. Kulekcioglu S, Sivrioglu K, Ozcan O and Parlak M. Effectiveness of low-level laser therapy in temporomandibular disorder Scand J Rheumatol (2003) 32:114-118.

15. Bezuur NJ, Habeets LLMH and Hansson TL. The effect of therapeutic laser treatment on patients with craniomandibular disorders. J Craniomandib Disord (1988) 2:83-86. 